Original papers

\title{
A taste sensor device for unmasking admixing of rancid or winey-vinegary olive oil to extra virgin olive oil
}

\author{
Ussama Harzalli $^{\mathrm{a}, \mathrm{b}}$, Nuno Rodrigues ${ }^{\mathrm{a}, \mathrm{c}}$, Ana C.A. Veloso ${ }^{\mathrm{d}, \mathrm{e}}$, Luís G. Dias ${ }^{\mathrm{a}, \mathrm{f}}$, José A. Pereira ${ }^{\mathrm{a}}$, \\ Souheib Oueslati ${ }^{\mathrm{b}}$, António M. Peres ${ }^{\mathrm{a}, \mathrm{g}, *}$

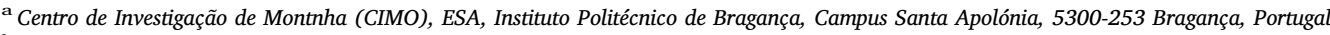 \\ ${ }^{\mathrm{b}}$ Institut Préparatoire aux Etudes Scientifiques et Techniques (IPEST), Laboratoire Materiaux, Molécules et Applications (LMMA), BP 51 La Marsa 2070, Tunisia \\ c Universidad de Léon, Departamento de Ingeniería Agrária, Av. Portugal, $n^{\circ}$ 41, 24071 Léon, Spain \\ d Instituto Politécnico de Coimbra, ISEC, DEQB, Rua Pedro Nunes, Quinta da Nora, 3030-199 Coimbra, Portugal \\ e CEB - Centre of Biological Engineering, University of Minho, Campus de Gualtar, 4710-057 Braga, Portugal \\ ${ }^{\mathrm{f}} \mathrm{CQ}$-VR, Centro de Química - Vila Real, UTAD, Apartado 1013, 5001-801 Vila Real, Portugal \\ ${ }^{g}$ Laboratory of Separation and Reaction Engineering - Laboratory of Catalysis and Materials (LSRE-LCM), ESA, Instituto Politécnico de Bragança, Campus Santa \\ Apolónia, 5300-253 Bragança, Portugal
}

\section{A R T I C L E I N F O}

\section{Keywords:}

Electronic tongue

Chemometrics

Olive oil

Physicochemical analysis

Sensory analysis

Adulteration detection

\begin{abstract}
A B S T R A C T
Electrochemical sensor devices have gathered great attention in food analysis namely for olive oil evaluation. The adulteration of extra-virgin olive oil with lower-grade olive oil is a common worldwide fraudulent practice, which detection is a challenging task. The potentiometric fingerprints recorded by lipid polymeric sensor membranes of an electronic tongue, together with linear discriminant analysis and simulated annealing metaheuristic algorithm, enabled the detection of extra-virgin olive oil adulterated with olive oil for which an intense sensory defect could be perceived, specifically rancid or winey-vinegary negative sensations. The homemade designed taste device allowed the identification of admixing of extra-virgin olive oil with more than $2.5 \%$ or $5 \%$ of rancid or winey-vinegary olive oil, respectively. Predictive mean sensitivities of $84 \pm 4 \%$ or $92 \pm 4 \%$ and specificities of $79 \pm 6 \%$ or $93 \pm 3 \%$ were obtained for rancid or winey-vinegary adulterations, respectively, regarding an internal-validation procedure based on a repeated K-fold cross-validation variant $(4$ folds $\times 10$ repeats, ensuring that the dataset was forty times randomly split into 4 folds, leaving $25 \%$ of the data for validation purposes). This performance was satisfactory since, according to the legal physicochemical and sensory analysis, the intentionally adulterated olive oil with percentages of $2.5-10 \%$, could still be commercialized as virgin olive oil. It could also be concluded that at a 5\% significance level, the trained panelists could not distinguish extra-virgin olive oil samples from those adulterated with $2.5 \%$ of rancid olive oil or up to $5 \%$ of winey-vinegary olive oil. Thus, the electronic tongue proposed in this study can be foreseen as a practical and powerful tool to detect this kind of worldwide common fraudulent practice of high quality olive oil.
\end{abstract}

\section{Introduction}

Olive oil quality classification as extra-virgin (EVOO), virgin (VOO) or lampante (LOO) olive oil is regulated by the European Union Commission (EU No 61/2011, 2011; EU No 1348/2013, 2013). These regulations take into account the legal levels defined for physicochemical parameters (e.g., free acidity, peroxide value, UV extinction coefficients and alkyl esters content), as well as, for positive and negative sensory sensations such as, the perception and the intensity of fruity positive attribute and the presence/absence of sensory defects (e.g., fusty, musty, rancid, winey-vinegary) (Borràs et al., 2015, 2016a, 2016b; Di Serio et al., 2017). Olive oil is highly appreciated by worldwide consumers due to the recognized health and nutritional benefits. Adulteration, frauds and mislabeling of olive oil have become a worldwide phenomenon leading to the decrease of the confidence of consumers (Jolayemi et al., 2017). Thus, different analytical techniques have been developed to detect olive oil adulterations (e.g., MALDITOF/MS technique; mid infrared, Raman, fluorescence or visible spectroscopy; DNA-targeted approaches; ion mobility spectrometry; nuclear magnetic resonance; dielectric technique; ultrasounds technique; gas chromatography; etc.), namely to identify and/or quantify the addition of other vegetable oils like camellia, canola, corn, grapeseed, hazelnut,

\footnotetext{
* Corresponding author at: Centro de Investigação de Montnha (CIMO), ESA, Instituto Politécnico de Bragança, Campus Santa Apolónia, $5300-253$ Bragança, Portugal.

E-mail address: peres@ipb.pt (A.M. Peres).
} 
peanut, rapeseed, soya, sesame, soybean and sunflower oils (De Melo Milanez and Pontes, 2015; Sun et al., 2015; Alouache et al., 2016; Jabeur et al., 2016; Kalaitzis and El-Zein, 2016; Nigri and Oumeddour, 2016; Mu et al., 2016; Rashvand et al., 2016; Srigley et al., 2016; Farley et al., 2017; Georgouli et al., 2017; Jergović et al., 2017; Liu et al., 2017; Ok, 2017; Philippidis et al., 2017; Santos et al., 2017; Uncu et al., 2017 ) or the admixture of lower quality or refined olive oils (Nigri and Oumeddour, 2016; Jergović et al., 2017). Although EVOO have a long history of economic adulteration, its detection still is a challenging task due to the diverse composition of cultivars and the limitations of existing detection methods (Ou et al., 2015; Srigley et al., 2016). The broad use of sensor-based devices, like electronic noses (E-noses) or electronic tongues (E-tongues), for olive oil sensory evaluation or olive oil discrimination based on the olive cultivar and geographical origin has been recently reviewed by Peris and Escuder-Gilabert (2016) and Valli et al. (2016). The literature survey clearly point out the limited number of works reporting the successful use of E-noses (Oliveros et al., 2002; Mildner-Szkudlarz and Jeleń, 2008, 2010; Lerma-García et al., 2010; Santonico et al., 2015) to detect olive oil adulteration with other vegetable oils or lower quality olive oils (possessing or not common sensory defects), as well as the scarce use of voltammetric E-tongues (Apetrei and Apetrei, 2014; Santonico et al., 2015). Recently, the use of a pontentiometric E-tongue device comprising cross-sensitivity lipid polymeric membranes, has demonstrated to be a practical and helpful taste sensor tool for olive oil analysis (Dias et al., 2014, 2016; Veloso et al., 2016, 2018; Slim et al., 2017; Souayah et al., 2017). It was previously reported by Marx et al. (2017b) and Slim et al. (2017) the capability of this type of E-tongue to provide quantitative potentiometric responses towards aldehydes, alcohols and esters compounds that mimic positive olive oil sensory attributes namely, 4-hydroxy-3methoxybenzaldehyde (vanilla sensation), hexyl acetate (sweet, green, grassy, fruity or apple sensations), (Z)-hex-3-en-1-ol (green leaves or banana sensations), (E)-hex-2-enal (green, almonds or apple sensations), (Z)-hex-3-enyl acetate (fruity or green leaves sensations), citric and tartaric acids (acid sensation), caffeine and quinine (bitter sensations) and sodium or potassium chloride (salty sensation). On the other hand, for negative sensations, Marx et al. (2017a) also described the quantitative responses towards n-butyric acid (butyric defect), 2-mercaptoethanol (putrid defect) and cyclohexanecarboxylic acid (zapateria defect). The sensing mechanism is dependent on the non-uniform hydrophilicity of the lipid membranes and on the ionic environment at the proximity of the membrane surface. Thus, the measured electric potential depends on the membrane surface-charge density changes, and on its permeability to ions altered by the physical adsorption of nonelectrolytes compounds (Iiyama et al., 1986; Kurihara et al., 1986; Hayashi et al., 1989). Recently Veloso et al. (2018) reported the capability of using an E-tongue device to classify olive oil according to the main sensory defect perceived. In this work, a pontentiometric E-tongue device was applied, for the first time, for detecting intentionallyadulterated EVOO with known percentages of rancid or winey-vinegary LOO (LOO-R or LOO-WV), which sensory defect and intensities were assessed by trained panelists.

\section{Materials and methods}

\subsection{Olive oil samples, physicochemical and sensory analysis}

Olive oils, produced from olives of Arbequina variety, were kindly supplied by a local olive oil producer of the Trás-os-Montes region (Macedo de Cavaleiros, Portugal). Fifteen liters of a high quality olive oil (EVOO) and ten liters of two types of low quality olive oil (LOO) were used. The LOO samples were intentionally chosen after ensuring that rancid (LOO-R, $5 \mathrm{Ls}$ ) or winey-vinegary (LOO-WV, $5 \mathrm{Ls)}$ negative sensations could be easily perceived by a sensory panel due to their high intensities. For the experiment, intentionally-adulterated EVOO olive oil samples were prepared and used, obtained by adding EVOO samples with pre-established volumes of LOO, resulting in volumetric adulterations (v/v) of the EVOO with $2.5 \%, 5 \%, 10 \%, 20 \%$ and $40 \%$ of LOO-R or LOO-WV. In total, 6 glass amber bottles of $250 \mathrm{~mL}$ each, were prepared for each adulteration level $(2.5-40 \%$, for each organoleptic defect, plus the negative (EVOO, i.e., $0 \%$ of adulteration) and positive (LOO-R or LOO-WV) controls. The established volumetric percentage levels took into account the fact that a $10 \%$ level of adulteration is high enough to be economically profitable but low enough to pass undetected (Srigley et al., 2016). Olive oil samples (EVOO, LOO-R, LOO$\mathrm{WV}$ and respective adulterated olive oil) were kept in amber bottles protected from the direct light exposition during 2 weeks before being used. To check the quality of the EVOO, LOO-R, LOO-WV and the intentionally-adulterated olive oil, all samples were subjected to physicochemical and sensory analysis, following the EU standard methods (EU No 61/2011, 2011; EU No 1348/2013, 2013). Five physicochemical quality parameters were evaluated: free acidity (FA, in\% oleic acid), the peroxide value (PV, in $\mathrm{mEq} \mathrm{O}_{2} / \mathrm{kg}$ ) and the specific coefficients of extinction at $232 \mathrm{~nm}$ and $270 \mathrm{~nm}\left(K_{232}\right.$ and $K_{270}$ and $\left.\Delta K\right)$. From each olive oil sample $(n=6)$, three independent sub-samples were collected and all physicochemical and chemical assays were carried out in triplicate. Each sample was also evaluated by eight trained panelists from the olive oil sensory panel of the School of Agriculture of the Polytechnic Institute of Bragança (Portugal), which was instructed by the panel leader about the type of defect that might be perceived or not in each sample (i.e., rancid or winey-vinegary sensations). The intensity of the positive or negative attributes perceived were graded according to an intensity scale ranging from 0 (no sensory sensation perceived) to 10 (maximum intensity of the sensory sensation perceived). Furthermore, for the final olive oil' quality grade classification the median intensities were used. The quality grade of the samples (EVOO, LOO and adulterated olive oil) was set considering the physicochemical levels and the sensory data (EU No 61/2011, 2011; EU No 1348/2013, 2013; IOC, 2013, 2014): EVOO (FA $\leq 0.8 \%$ oleic acid, $\mathrm{PV} \leq 20 \mathrm{mEq} \mathrm{O}_{2} / \mathrm{kg}, \quad K_{232} \leq 2.50, \quad K_{270} \leq 0.22, \Delta K \leq 0.01 ;$ fruity median intensity greater than 0 and median intensity of defects equal to 0 ); VOO (FA $\leq 2.0 \%$ oleic acid, $\mathrm{PV} \leq 20 \mathrm{mEq} \mathrm{O}_{2} / \mathrm{kg}, K_{232} \leq 2.60$, $K_{270} \leq 0.25, \Delta K \leq 0.01$; fruity median intensity greater than 0 and median intensity of defects greater than 0 and lower than 3 ) or LOO (in the other cases). All assays were performed at the laboratories of the School of Agriculture - Polytechnic Institute of Bragança (Portugal).

\subsection{E-tongue}

\subsubsection{E-tongue device and set-up}

The E-tongue multi-sensor device (Fig. 1) included two homemade print-screen potentiometric arrays covered with an acrylic resin (PLASTIK 70) for ensuring a waterproof surface. As previously described (Dias et al., 2015; Veloso et al., 2018), each polyvinyl chloride (PVC) board $(3 \mathrm{~cm} \times 12 \mathrm{~cm})$ had 20 wells $(3.6 \mathrm{~mm}$ of diameter and $0.3 \mathrm{~mm}$ of thickness), where 20 cross-sensitivity lipid polymeric membranes were applied, using a drop-by-drop technique. The polymeric membranes had different combinations of 4 lipid additives $(\sim 3 \%$ : octadecylamine, oleyl alcohol, methyltrioctylammonium chloride or oleic acid), 5 plasticizers ( $\sim 32 \%$ : bis(1-butylpentyl) adipate, dibutyl sebacate, 2-nitrophenyl-octylether, tris(2-ethylhexyl)phosphate or dioctyl phenylphosphonate) and PVC ( $\sim 65 \%)$, which were used as chemical sensors (Fluka, minimum purity $\geq 97 \%$ ). Even if the two E-tongue arrays comprised sensor membranes with the same lipid additive/plasticizer/PVC mixture and with the same relative composition, they showed different electrochemical properties, which could be attributed to the formation of inhomogeneous membranes with different physical properties (e.g., different membrane transparency levels and porosity leading to different adsorption phenomena and surface chemical reactions, which may lead to deviations in sensors' readings). Therefore, it was considered that the device comprised 40 independent sensors instead of assuming a set of 20 sensor-sensor replica membranes. At the 


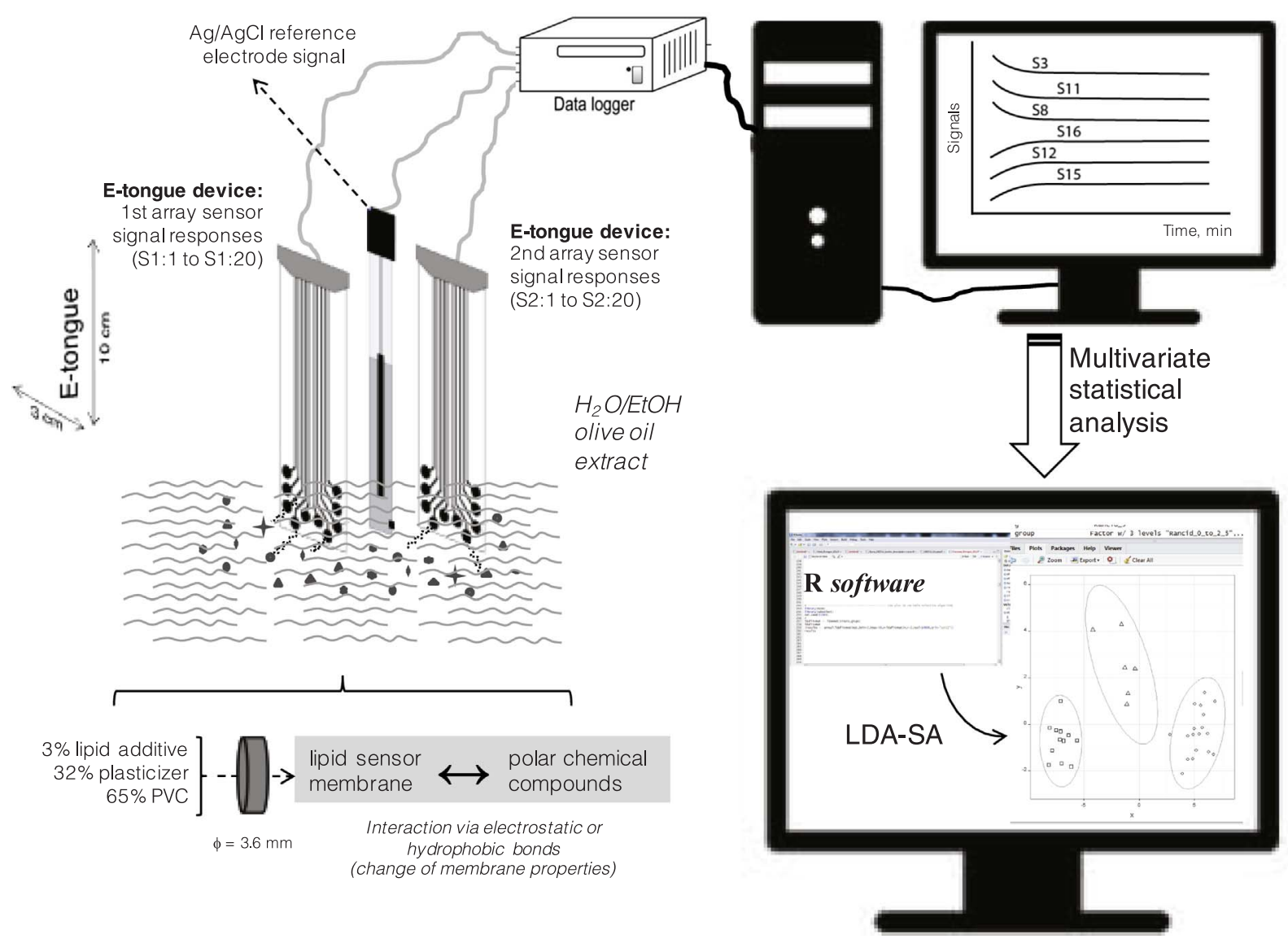

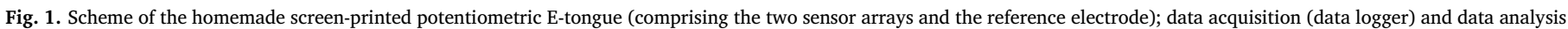
(R software) set-up; with dimensions, generic sensor membranes composition and interaction mechanisms.

end of the PVC board, a RS-232 plug (20 pins) was placed, allowing connecting each lipid sensor membrane through a printed scheme containing silver epoxy conducting resin (EPO-TEK E4110) to a multiplexer Agilent Data Acquisition Switch Unit model 34970A, controlled with the Agilent BenchLink Data Logger software installed on a PC (Fig. 1). Each potentiometric cycle measurement took $5 \mathrm{~min}$ and allowed recording the multi-sensor device signal profiles that corresponded to the potential generated between each of the 40 sensor membranes (due to the change of the membrane properties by the electrostatic or hydrophobic interactions (Kobayashi et al., 2010) established between the membrane and the polar chemical compounds present in the hydro-ethanolic olive oil extracts) and an $\mathrm{Ag} / \mathrm{AgCl}$ double-junction glass electrode used as the reference electrode (Crison, 5241). The multi-sensor E-tongue device was stored in a $\mathrm{KCl}$ aqueous solution $(1 \mathrm{~mol} / \mathrm{L})$. The same sensor coding used previously was also adopted and so each sensor was identified with a letter $\mathrm{S}$ (for sensor) followed by the number of the array ( 1 or 2 ) and the number of the membrane (1-20, corresponding to different combinations of plasticizer and additive used) (Veloso et al., 2018).

\subsubsection{E-tongue analysis: Olive oil sample preparation and potentiometric} assays

All samples were electrochemically analyzed within the same day avoiding the need of statistical complex signal pre-treatments to overcome possible potential drifts (Dias et al., 2009; Marx et al., 2017b; Veloso et al., 2018). The olive oil samples were extracted using a previous described procedure (Dias et al., 2014). Samples $(10 \mathrm{~g})$ were withdrawn from each olive oil bottle (non adulterated or intentionallyadulterated olive oils) after being smoothly shaken, and then mixed with $100 \mathrm{~mL}$ of water-ethanol solutions $(80: 20, \mathrm{v} / \mathrm{v})$ during 5-10 min using a vortex stirrer (LBX V05 series, lbx instruments) at $500 \mathrm{rpm}$. The mixture was left at ambient temperature during $60 \mathrm{~min}$, after which, $40 \mathrm{~mL}$ of the supernatant solution was carefully removed and immediately analyzed with the E-tongue. The electrochemical analysis took 5 min enabling to carry out several electrochemical scans, being retained the last one, which would correspond to a pseudo-equilibrium state. Electrochemical assays were performed in duplicate for each sample unless the coefficients of variation of the potentiometric signals recorded by each E-tongue sensor were greater than $20 \%$ (value set according to the IOC regulations for sensory analysis), in which cases a third assay was performed. As proposed by Rodrigues et al. (2016), to minimize the risk of overoptimistic performance of the multivariate models, for data split (establishment of training and internal-validation sets) and modeling purposes, only one electrochemical "average" signal profile per sample was used, avoiding that results from duplicate assays of the same olive oil sample could be included into both training and validation sets. Ethanol was of analytical grade (Panreac, Barcelona) and deionized water was of type II.

\subsection{Statistical analysis}

One-way analysis of variance (one-way ANOVA) was applied to evaluate the existence of statistical significant differences among physicochemical mean levels or mean intensities of the negative sensory attributes evaluated in EVOO adulterated or not with LOO-R or LOOWV samples (adulteration levels: 0\%, 2.5\%, 5\%, 10\%, 20\%, 40\% and/ or $100 \%)$. When a statistical significant difference was detected, the pos-hoc multi-comparison Tukey's test was further used to identify the differences among adulteration levels. Linear Pearson correlation coefficients ( $R$-Pearson) were calculated to evaluate the existence of bivariate correlations between physicochemical parameters or negative sensory attributes with the olive oil adulteration percentage. Finally, 
the potentiometric E-tongue capability to detect adulterated EVOO due to the intentionally addition of different percentages of LOO-R or LOO$\mathrm{WV}$, was evaluated using linear discriminant analysis (LDA) coupled with the meta-heuristic simulated annealing (SA) variable selection algorithm (Bertsimas and Tsitsiklis, 1992; Kirkpatrick et al., 1983; Cadima et al., 2004). The electrochemical-chemometric strategy followed was similar to previous works of the research team (Dias et al., 2016; Rodrigues et al., 2016; Veloso et al., 2016, 2018; Marx et al., 2017a, 2017b, 2017c; Slim et al., 2017; Souayah et al., 2017). The signal profiles generated during the electrochemical analysis of the hydro-ethanolic extracts of non-adulterated and intentionally-adulterated olive oils were subjected to a linear discriminant analysis (LDA) in combination with a meta-heuristic simulated annealing (SA) algorithm, in order to establish predictive E-tongue-LDA-SA models capable of classifying olive oil according to their adulteration level. This variable selection algorithm was used aiming to identify the most informative sub-set of sensors (which could include from 2 to a maximum of 39 sensors, used as independent predictors) for each type of EVOO sensory adulteration studied (i.e., EVOO intentionally-adulterated with different levels of LOO-R or LOO-WV), avoiding the inclusion of redundant sensors' information, which may increase noise effects. The LDA predictive performance was evaluated using two cross-validation (CV) variants: leave-one-out (LOO-CV), known to be an over-optimistic procedure; and, repeated K-fold (repeated K-fold-CV) technique. For the latter, data was randomly split into $\mathrm{K}$ folds, being each of the folds left out in turn and the other K-1 folds used to train the model. The held out fold was used for test purposes and the quality of the predictions was assessed using the average values of sensitivities (percentage of correct/ true classifications) and specificities (assumed as the true negative rates). The $\mathrm{K}$ estimates are averaged to get the overall resampled estimate (Kirkpatrick et al., 1983). In this work, the K-folds were set equal to 4 , enabling the random formation of internal validation subsets (for each gustatory group) with $25 \%$ of the initial data, allowing bias reduction. The procedure was repeated 10 times for putting the model under stress. The repeated K-fold-CV technique allows reducing the uncertainty of the estimates, by evaluating the predictive performance of the models established using $4 \times 10$ random sub-sets for internal validation (i.e., 40 total resamples). To normalize the weight of each variable in the final linear classification model, variable scaling and centering procedures were evaluated. The classification performance of each LDA model was graphically evaluated using 2-D plot of the main discriminant functions (when more than two class groups were considered). For the multi-classes case, posterior probabilities were computed using the Bayes' theorem (which enables controlling over-fitting issues) to deeper assess the classification capability of the established LDA models (i.e., to infer the probability obtained after an event has been observed), being also plotted as the class membership boundary lines in the 2-D plots (Bishop, 2006). All statistical analysis were performed using the Subselect (Cadima et al., 2004, 2012; Kuhn and Johnson, 2013) and MASS (Venables and Ripley, 2002) packages of the open source statistical program $\mathrm{R}$ (version 2.15 .1 ), at a $5 \%$ significance level.

\section{Results and discussion}

\subsection{Olive oil quality grade classification and effect of the adulteration level} on physicochemical and sensory data

Physicochemical quality parameters (FA, PV, $K_{232}, K_{270}$ and $\Delta K$ values) were assessed for each type of olive oil sample (EVOO, LOO or adulterated olive oil) as well as the intensity of each sensory negative attribute (i.e., rancid or WV), being the results shown in Tables 1 and 2, for intentionally-adulterations with LOO-R or LOO-WV, respectively. The negative attributes winey-vinegary and rancid were studied in this work since they are the two most common sensory defects detected in olive oils (Morales et al., 2005; Cayuela et al., 2015). Winey-vinegary is characteristic of certain olive oils and is related to the formation of acetic acid, ethyl acetate and/or ethanol due to the fermentation of olives during storage before olive oil extraction. Rancid is characteristic of olive oils that undergo oxidation.

Olive oil quality grade classification (adulterated or not) was established based on the legal thresholds for physicochemical and sensory data, being for the latter used the median intensity of the defect mostly perceived, following the EU and IOC guidelines (EU No 61/2011, 2011; EU No 1348/2013, 2013; IOC, 2013, 2014). However, since the panelists assessed defect intensities using a continuous rule scale $(0-10 \mathrm{~cm}$, corresponding to $0-10$ scale of intensity sensation perception), instead of an ordinary scale, in this work, the mean intensity values of the negative attributes were also determined and further used for statistical analysis (one-way ANOVA followed by Tukey's HSD test), allowing to infer the effect of the adulteration percentage in the evaluated parameters.

Based on the results (Tables 1 and 2), it can be concluded that olive oil intentionally-adulterated with percentages from $2.5 \%$ to $10 \%$ of LOO-R or LOO-WV could still be commercialized as VOO, since the physicochemical and sensory quality legal requirements were fulfilled. Indeed, only EVOO samples adulterated with $20 \%$ or $40 \%$ would be classified as LOO. Furthermore, the statistical analysis showed that although the defect intensity (rancid or WV) was significantly affected by the adulteration level ( $P$-value $<.0001$, for the one-way ANOVA), it also pointed out that, from the overall sensory evaluation, the panelists could not easily assess low adulteration levels, being in general no significant statistical difference was found for the mean defect intensity perceived on non-adulterated (EVOO) and intentionally adulterated olive oil samples with $2.5 \%$ of LOO-R ( $P$-value $\geq .2502$, for Tukey's HSD test) or up to $10 \%$ with LOO-WV ( $P$-value $\geq .0759$, for Tukey's HSD test). Similarly, for the physicochemical parameters, in general, no significant statistical differences were found up to $5-10 \%$ adulteration levels ( $P$-value $>.0500$, for the Tukey's HSD test). Nevertheless, for the rancid intentionally-adulterated olive oil linear relationships $(R$ Pearson $\geq 0.9701$ ) could be observed between the physicochemical contents and the adulteration level (from $0 \%$ up to $100 \%$ ) as well as for the defect intensity perceived with the adulteration level $(R$ Pearson $=0.8661$, for $0 \%$ up to $40 \%$ adulteration). On the contrary, no such evident linear relationships were found relating the physicochemical levels and the adulteration percentage (0-100\%) for the olive oil adulterated with LOO-WV $(0.7751 \leq R$-Pearson $\leq 0.9692)$. In fact, this negative attribute is mainly related with a degradation processes that occurred in the olives and stopped at the extraction procedure, thus not affecting in high level the levels of the quality physicochemical parameters of the final product. Also, only a slight linear trend $(R$ Pearson $=0.7808$ ) was observed between the WV defect intensity and the adulteration level $(0-40 \%)$.

Actually, the higher difficulty of the sensory panel in detecting low levels of adulteration with LOO-WV compared to LOO-R was expected. Rancid defect is mainly due to the oxidation reactions that take place in the olive oil in the presence of oxygen, which are potentiated when some rancid already exist in the olive oil, being expected that the level of this negative attribute could be enhanced during olive oil storage, leading to high changes of the quality physicochemical parameters levels (namely, PV and UV-Vis extinction coefficients). On the other hand, the WV defect is mainly due to sugars' degradation processes and alcohol fermentation that might have occurred in the olives (due to the presence of microorganisms, probably yeasts). These phenomena should stop or decrease to a minimum level, and not affect in high degree the physicochemical parameters. Finally, in low levels, the perception of WV negative attribute can be confused with the sensation of grapevine related to olive oil extracted from ripe or overripe fruits. These findings pointed out the difficulty of assessing this latter olive oil fraudulent practice based on the typical physicochemical or sensory olive oil evaluation approaches and the need of fast and cost-effective alternatives based on analytical methodologies such as electrochemical 


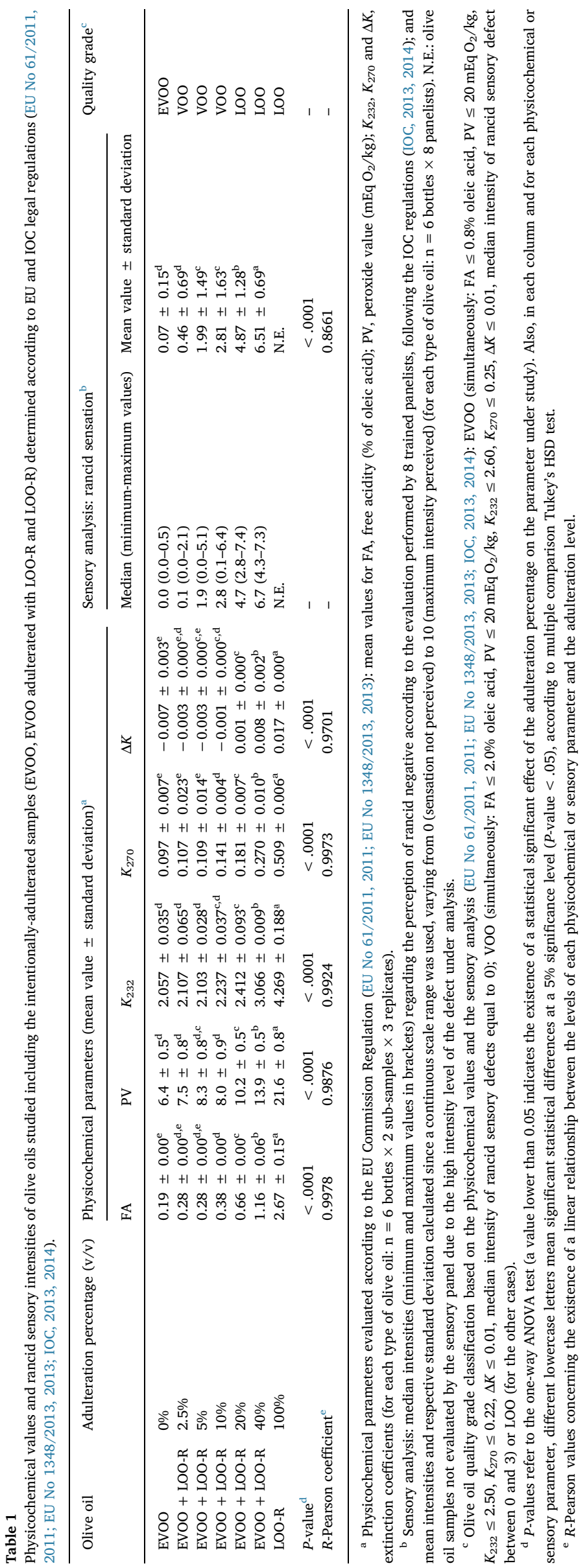




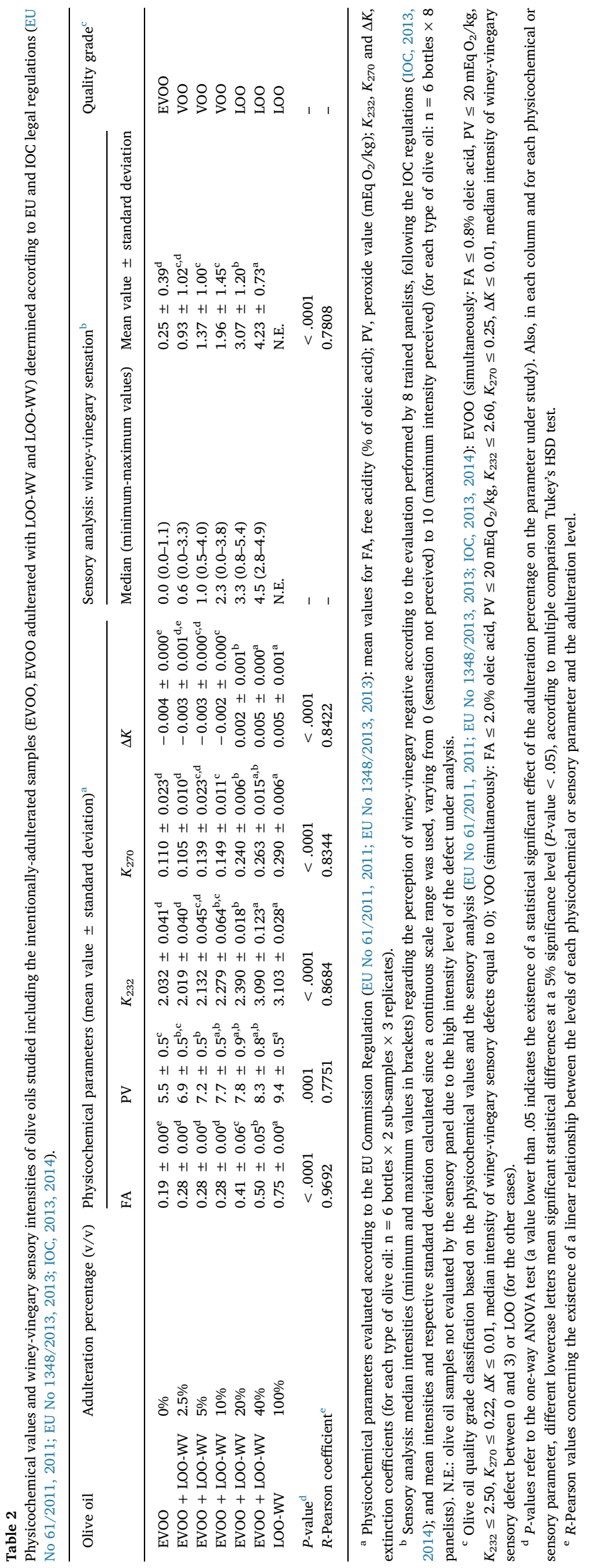


sensor devices.

3.2. Evaluation of non-adulterated and intentionally-adulterated EVOO with low quality olive oil (LOO-R and LOO-WV) using an E-tongue

The possible use of the potentiometric signal profiles gathered by the homemade E-tongue device during the analysis of the hydro-ethanolic extracts of the olive oil, as fingerprints for EVOO adulteration with low quality olive oil containing sensory defects (LOO-R or LOO$\mathrm{WV}$ ) was evaluated for the first time. Indeed, as already referred, in the literature only two studies addressed this challenging task (olive oil adulteration with low quality olive oil) using E-tongues, but in both works voltammetric devices were used (Apetrei and Apetrei, 2014; Santonico et al., 2015). Moreover, in the referred works, the adulteration detection due to the addition of different percentages of olive oil containing intense sensory defects was not addressed. Recently, a potentiometric E-tongue with lipid polymeric sensor membranes was successfully applied to classify commercial olive oil according to the main sensory defect perceived (Veloso et al., 2018).

\subsubsection{Olive oil potentiometric signal profiles}

Depending on the type of olive oil (i.e., EVOO not adulterated or EVOO intentionally-adulterated with different levels of LOO-R or LOO$\mathrm{WV}$ ), slight different potentiometric signal profiles were acquired by the E-tongue sensors (20 different lipid polymeric membranes in duplicate: S1:1-S1:20 and S2:1-S2:20) during the analysis of the olive oil' hydro-ethanolic extracts, as can be inferred from Fig. 2. In fact, the potential signals varied from -210 to $+340 \mathrm{mV}$ for EVOO (not adulterated), from -190 to $+400 \mathrm{mV}$ and -190 to $+490 \mathrm{mV}$ for adulterated olive oil (merging the considered five of adulteration levels) with LOO-R or LOO-WV, respectively. Moreover, the signal recorded by some of the 40 E-tongue lipid membranes linearly increased $(0.8504 \leq R$-Pearson $\leq 0.9938) \quad$ or decreased $\quad(-0.9992 \leq R$ Pearson $\leq-0.8717$ ) with the rancid adulteration level (in the range of $0-40 \%$ of LOO-R). For WV adulteration, when a trend was observed for the lipid membranes, the signal recorded increased linearly $(0.8504 \leq R$-Pearson $\leq 0.9935)$ with the percentage of WV adulteration (in the range of $0-20 \%$ of LOO-WV) followed, in general, by a signal decrease for the maximum adulteration level studied $(40 \%$ of LOO-WV). The overall analysis of the E-tongue signal profiles gathered, which showed the existence of signal trend differences (signal intensities and dynamic signal ranges) between non-adulterated and intentionally-adulterated olive oil, as well as linear E-tongue signal responses towards the adulteration levels, may foreseen the feasibility of using the potentiometric fingerprints as a possible analytical marker for the detection of EVOO adulteration with LOO-R or LOO-WV.

\subsubsection{Discrimination of non-adulterated EVOO and intentionally-} adulterated EVOO with LOO-R or LOO-WV

Recently, it was shown that a potentiometric E-tongue could be successfully used to classify olive oil according to the main sensory defect (i.e., fusty, rancid, wet-wood or winey-vinegary) (Veloso et al.,
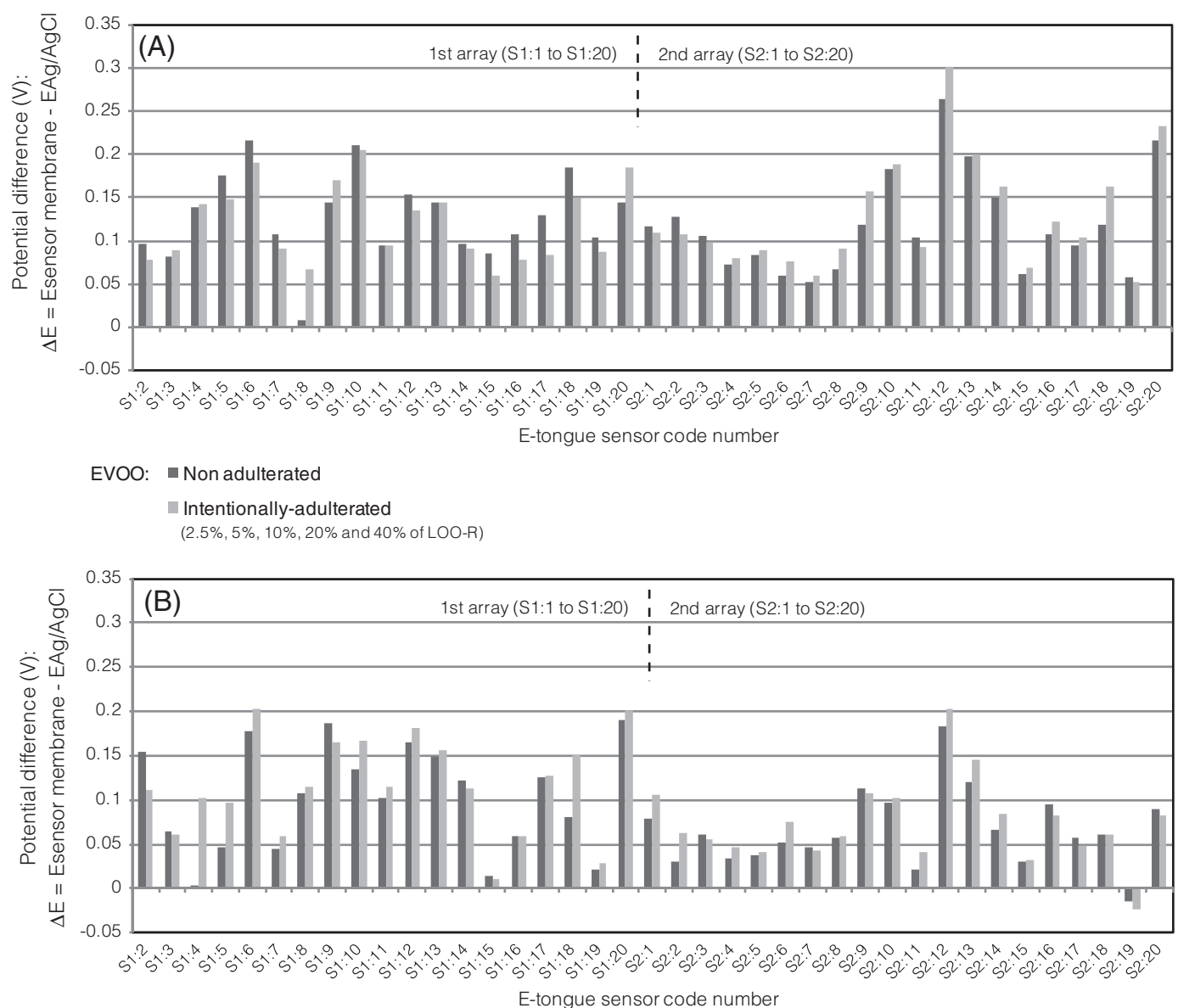

EVOO: Non adulterated

- Intentionally-adulterated

(2.5\%, $5 \%, 10 \%, 20 \%$ and $40 \%$ of LOO-WV)

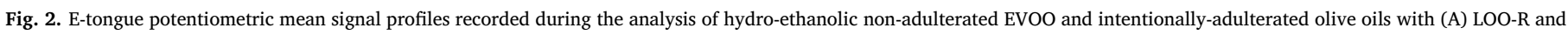
(B) LOO-WV. 
2018), confirming the competence of this type of taste sensor device to give qualitative or quantitative responses towards positive or negative sensory attributes usually found in olive oil (Veloso et al., 2016; Slim et al., 2017) or in table olives (Marx et al., 2017a, 2017c). Thus, in the present work, E-tongue-LDA-SA models were established based on selected sub-sets of sensors for discriminating non-adulterated EVOO or intentionally-adulterated EVOO with different levels of LOO-R or LOOWV $(2.5 \%, 5 \%, 10 \%, 20 \%$ or $40 \%$, in $\mathrm{v} / \mathrm{v})$. The linear multivariate models performances were assessed through the calculation of the respective sensitivities (i.e., percentages of correct classifications) and specificities (assumed as the true negative rates) for original grouped data and further confirmed for LOO-CV and repeated K-fold-CV procedures.

For adulterations with low quality rancid olive oil, three groups were pre-established; one for non-adulterated EVOO and adulterated with $2.5 \%$ of LOO-R (rancid adulteration $\leq 2.5 \%$ ), other including only adulterated EVOO with $5 \%$ of LOO-R ( $5 \%$ rancid adulteration) and the last group including adulterated EVOO with $10 \%, 20 \%$ and $40 \%$ of LOO-R $(10 \% \leq$ rancid adulteration $\leq 40 \%)$. Indeed, preliminary evaluations showed non-adulterated EVOO and adulterated EVOO with $2.5 \%$ of LOO-R could not be effectively distinguished, which is in agreement with the conclusions previously reached with the olive oil physicochemical and sensory analysis. Furthermore, for the abovementioned 3 groups (rancid adulteration $\leq 2.5 \%$; $5 \%$ rancid adulteration; and, $10 \% \leq$ rancid adulteration $\leq 40 \%$ ), the potentiometric signal data collected allowed to establish an E-tongue-LDA-SA model (2 discriminant functions explaining $95.9 \%$ and $4.1 \%$ of the original data variability) based on the signal profiles recorded from 19 sensor membranes of the E-tongue device (1st array sensors: S1:1, S1:2, S1:7, S1:9, S1:10, S1:11 to S1:13, S1:17 and S1:29; 2nd array sensors: S2:1, S2:5, S2:6, S2:9, S2:10, S2:13, S2:14, S2:17 and S2:19), during the analysis of olive oil' hydro-ethanolic extracts. The established model allowed the correct classification of all olive oil (adulterated or not) according to the pre-defined group (i.e., an overall sensitivity and specificity of $100 \%$ ) for the original grouped data (Fig. 3). As can be inferred from the observation of Fig. 3, the 1st discriminant function is capable of fully discriminating the 3 groups formed according to their increasing level of adulteration, showing a possible direct correlation between the increasing magnitude of the group centroids of the 1st LDA function and the increasing rancid adulteration level. For the LOO-CV procedure, only 3 of the 36 olive oil evaluated were misclassified, corresponding to a global sensitivity of $92 \%$ (with groups' sensitivities of $92 \%, 83 \%$ and $94 \%$ for rancid adulteration $\leq 2.5 \%, 5 \%$ rancid adulteration and $10 \% \leq$ rancid adulteration $\leq 40 \%$, respectively) and specificity of $87 \%$ (with groups' specificities of $79 \%, 83 \%$ and $100 \%$ for rancid adulteration $\leq 2.5 \%, 5 \%$ rancid adulteration and $10 \% \leq$ rancid adulteration $\leq 40 \%$, respectively). The individual specificities obtained showed that more than $79 \%$ of the correct classifications were not false predictions (true negative values) for the three olive oil groups considered. Furthermore, the E-tongue-LDA-SA predictive performance was assessed through the repeated K-fold-CV procedure ( 4 folds and 10 repetitions, which allowed the evaluation of the robustness of the model using the 40 internal cross-validation sets of $8-10$ olive oil, where each fold was used as external validation test, constituted of 3 samples of rancid adulteration $\leq 2.5 \%, 1$ or 2 samples of $5 \%$ rancid adulteration and 4 or 5 samples of $10 \% \leq$ rancid adulteration $\leq 40 \%$ ). The best predictive E-tongue-LDA-SA model was also based on the potentiometric fingerprints recorded by the same 19 lipid membrane sensors, which led to mean correct classification rates of $84 \pm 4 \%$ (varying from $79 \%$ to $94 \%$ for each of the 10 repeated split procedure, each constituted by 4 random folds) and an average specificity of $79 \pm 6 \%$ (ranging from $72 \%$ to $90 \%$ for each of the 10 random repetitions of the 4 folds data split). The obtained results with this more robust $\mathrm{CV}$ variant had similar effectiveness regarding the individual groups correct classification rates (sensitivities: $86 \pm 8 \%, 64 \pm 7 \%$ and $92 \pm 5 \%$ with related minimum posterior probabilities greater

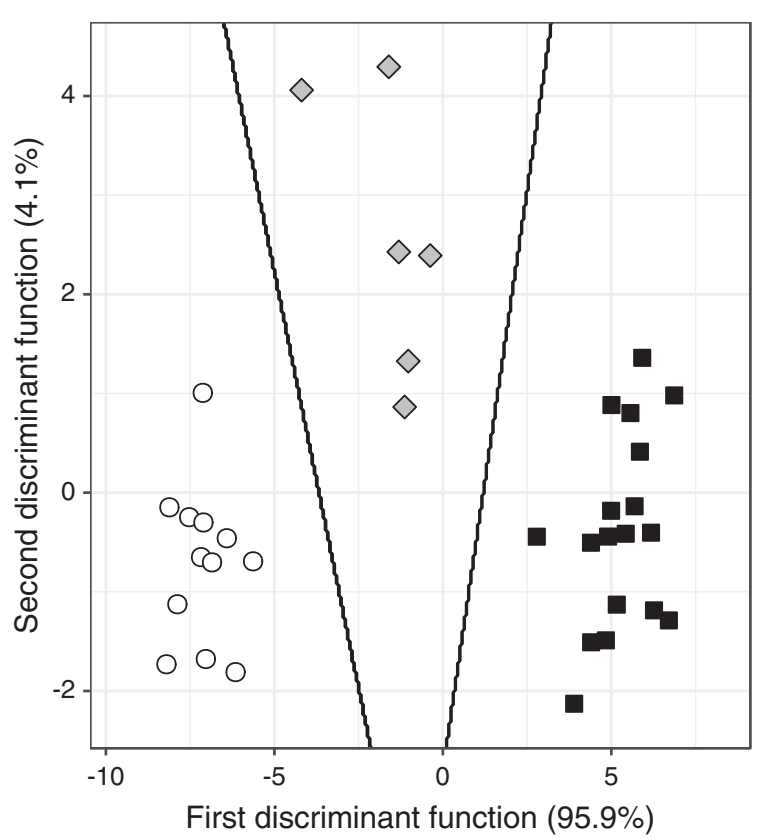

Rancid 0 to $2.5 \% \quad \diamond$ Rancid $5 \%$ Rancid 10 to $40 \%$

Fig. 3. E-tongue-LDA-SA model performance (original grouped data) in semi-quantitatively detecting EVOO adulterations with different levels (2.5-40\%, v/v) of a low-quality rancid olive oil, based on the potentiometric fingerprints recorded by 19 lipid membrane sensors, according to the predefined adulteration groups: rancid adulteration $\leq 2.5 \%$ (including not adulterated and $2.5 \%$ adulterated olive oils); $5 \%$ rancid adulteration; and, $10 \% \leq$ rancid adulteration $\leq 40 \%$.

than $72 \%, 58 \%$ and $87 \%$; and, specificities: $81 \pm 9 \%, 62 \pm 12 \%$ and $93 \pm 5 \%$; for olive oils belonging, respectively, to rancid adulteration $\leq 2.5 \%, 5 \%$ rancid adulteration and $10 \% \leq$ rancid adulteration $\leq 40 \%$ olive oil adulteration groups), which strengthen the possibility of using the potentiometric E-tongue device to semiquantitatively detect adulteration ranges of EVOO with lower quality olive oil possessing an intense rancid defect (LOO-R). Nevertheless, it should be remarked that, from the results of the repeated K-fold-CV, it is clear that EVOOs with $5 \%$ rancid adulteration may be easily misclassified as belonging to one of the other olive oil adulteration groups. So, the potentiometric E-tongue would be a more feasible tool for identifying rancid adulteration levels greater than $5 \%$, thus with a performance slightly lower than that shown by the sensory panel (which, based on the ANOVA results, could discriminate rancid adulteration levels greater than $2.5 \%$ ).

Similarly, based on preliminary evaluations of adulterations with low quality winey-vinegary olive oil, 3 groups were also pre-established; one grouping non-adulterated EVOO and adulterated with $2.5 \%$ of LOO-WV (WV adulteration $\leq 2.5 \%$ ), other including adulterated EVOO with $5 \%$ and $10 \%$ of LOO-WV $(5 \% \leq$ WV adulteration $\leq 10 \%)$ and the last group including adulterated EVOO with $20 \%$ and $40 \%$ of LOO-WV $(20 \% \leq \mathrm{WV}$ adulteration $\leq 40 \%)$. An E-tongue-LDA-SA model, with 2 discriminant functions (explaining $99.1 \%$ and $0.9 \%$ of the original data variability) based on the potentiometric signals of 20 E-tongue sensors (1st array sensors: S1:2, S1:3, S1:6, S1:13 to S1:15 and; 2nd array sensors: S2:1, S2:2, S2:6 to S2:14, S2:18 and S2:19) was selected. The supervised multivariate linear model enabled the correct classification of the 36 olive oils (adulterated or not) according to the pre-defined olive oil group, with $100 \%$ overall sensitivity and specificity for the original grouped data (Fig. 4), as well as for the LOO-CV procedure. From the visualization of Fig. 4, it is also evident that the 1st discriminant function allowed a complete discrimination of the 3 groups formed according to their increasing level of adulteration, showing a probable direct correlation between the increasing magnitude of the 1st discriminant function group centroids and the increasing 


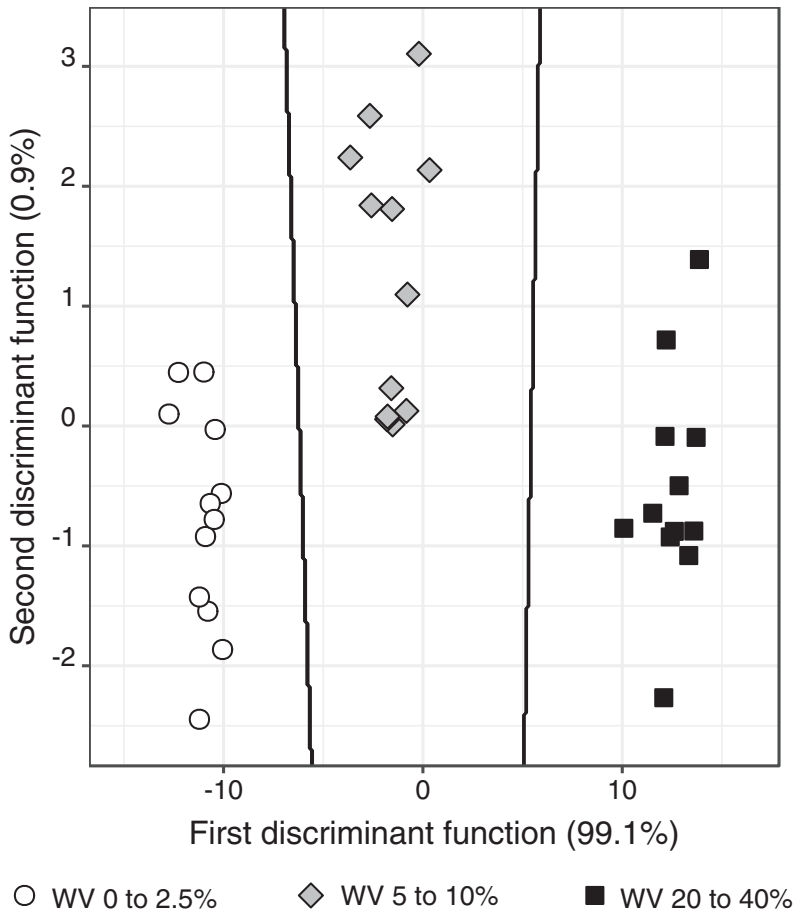

Fig. 4. E-tongue-LDA-SA model performance (original grouped data) in semi-quantitatively detecting EVOO adulterations with different levels $(2.5-40 \%, \mathrm{v} / \mathrm{v})$ of a low-quality winey-vinegary olive oil, based on the potentiometric fingerprints recorded by 20 lipid membrane sensors, according to the predefined adulteration groups: WV adulteration $\leq 2.5 \%$ (including not adulterated and $2.5 \%$ adulterated olive oils); $5 \% \leq \mathrm{WV}$ adulteration $\leq 10 \%$; and, $20 \% \leq \mathrm{WV}$ adulteration $\leq 40 \%$.

WV adulteration level. The E-tongue-LDA-SA predictive performance was further evaluated using the repeated K-fold-CV procedure ( 4 folds and 10 repetitions, which allowed the evaluation of the robustness of the model using 40 internal cross-validation sets of 9 olive oil samples, where each set was used as external validation sets, constituted by 3 samples of all WV adulteration groups considered). The results also showed that the best predictive E-tongue-LDA-SA model was based on the potentiometric data recorded by the same 20 sensors, allowing obtaining mean correct classification rates of $92 \pm 4 \%$ (varying from $89 \%$ to $100 \%$ for each of the 10 repeated split procedure, each constituted by 4 random folds) and an average specificity of $93 \pm 3 \%$ (ranging from $89 \%$ to $100 \%$ for each of the 10 random repetitions of the 4 folds data split). For the individual groups, correct classification rates of the same magnitude were obtained (sensitivities: $92 \pm 6 \%$, $92 \pm 7 \%$ and $93 \pm 3 \%$ with related minimum posterior probabilities greater than $65 \%, 64 \%$ and $75 \%$; with specificities: $94 \pm 6 \%$, $87 \pm 7 \%$ and $96 \pm 7 \%$; for olive oil belonging, respectively, to WV adulteration $\leq 2.5 \%, 5 \% \leq \mathrm{WV}$ adulteration $\leq 10 \%$ and $20 \% \leq \mathrm{WV}$ adulteration $\leq 40 \%$ olive oil adulteration groups), showing the feasibility of applying the potentiometric E-tongue to semi-quantitatively detect adulteration ranges of EVOO with lower quality olive oil possessing an intense WV defect (LOO-WV). For adulterations with WV defect, the E-tongue was able to identify adulteration levels greater than $2.5 \%$, showing a superior performance compared to that achieved by the taste sensor device for adulterations with rancid defect or even by the sensory panel, which results pointed out (based on the ANOVA results) a trend to give statistical similar defect intensities for olive oil without adulteration and adulterated with $2.5 \%$ or $5 \%$ of LOO-WV (and in some cases up to $10 \%$ of adulteration).

Finally, it should be remarked that the predictive performance of the homemade potentiometric E-tongue, used for the first time for identifying EVOO adulteration with low quality rancid or winey-vinegary olive oil (LOO-R or LOO-WV), was as satisfactory as those reported with voltammetric E-tongue devices. Apetrei and Apetrei (2014) showed that a voltammetric E-tongue could be used correctly to classify adulterated olive oil when the concentration level of adulterant oil (corn, soybean or sunflower oils) was between $5 \%$ and $10 \%$. Also, Santonico et al. (2015), reported the capability of a voltammetric Etongue to detect the presence of fraudulent admixing of $5 \%$ of extraneous vegetable oils (e.g., soybean, sunflower seeds and peanut oils), although this detection limit would increase up to $8 \%$ if the admixing was with pomace oil.

\section{Conclusions}

The work carried out confirmed that physicochemical quality parameters, usually required for establishing the quality grade of an olive oil, could not be used to detect intentionally admixing of low quality rancid or winey-vinegary olive oil to extra virgin olive oil, allowing a classification of virgin olive oil to EVOO with adulteration levels up to $20 \%$. The sensory analysis presented a more efficient tool, restricting virgin olive oil classification to admixing up to $10 \%$ and being able to detect adulterations greater than $2.5 \%$ or $5 \%$, depending if adulteration was made with rancid or winey-vinegary olive oil, respectively. Considering the inherent constrains for olive oil classification (scarcity of trained panels, experts' subjectivity, lack of reference standards, time consuming analyses), the possibility of using the proposed homemade potentiometric E-tongue for detecting admixing rancid or winey-vinegary olive oil at low levels (as small as $2.5-5 \%$ of adulteration), is of utmost relevance for guarantee the high quality olive oil. Finally, the work is giving an important contribution in demonstrating the broad potential of potentiometric multi-sensor arrays to be used as a complementary artificial analytical sensory device within the olive oil analysis.

\section{Ethical approval}

This article does not contain any studies with human participants or animals performed by any of the authors.

\section{Informed consent}

Not applicable.

\section{Acknowledgments}

This work was financially supported by Project POCI-01-0145FEDER-006984 - Associate Laboratory LSRE-LCM, Project UID/QUI/ 00616/2013 - CQ-VR, Project UID/BIO/04469/2013 - CEB and strategic project PEst-OE/AGR/UI0690/2014 - CIMO all funded by FEDER - Fundo Europeu de Desenvolvimento Regional through COMPETE2020 - Programa Operacional Competitividade e Internacionalização (POCI) - and by national funds through FCT - Fundação para a Ciência e a Tecnologia, Portugal. Nuno Rodrigues thanks FCT, POPH-QREN and FSE for the Ph.D. Grant (SFRH/BD/104038/2014). Souheib Oueslati is also grateful for the support of the Tunisian Ministry of Agriculture.

\section{Conflict of interest}

None.

\section{References}

Alouache, B., Boutkedjirt, T., Laux, D., 2016. Ultrasonic assessment of olive oil mixtures properties. In: IEEE International Ultrasonics Symposium, IUS, 2016-November, 7728604.

Apetrei, I.M., Apetrei, C., 2014. Detection of virgin olive oil adulteration using a voltammetric e-tongue. Comput. Electron. Agric. 108, 148-154.

Bertsimas, D., Tsitsiklis, J., 1992. Simulated annealing. Stat. Sci. 8, 10-15.

Bishop, C.M., 2006. Pattern Recognition and Machine Learning, first ed. Springer, New 
York

Borràs, E., Ferré, J., Boqué, R., Mestres, M., Aceña, L., Calvo, A., Busto, O., 2016a. Olive oil sensory defects classification with data fusion of instrumental techniques and multivariate analysis (PLS-DA). Food Chem. 203, 314-322.

Borràs, E., Ferré, J., Boqué, R., Mestres, M., Aceña, L., Calvo, A., Busto, O., 2016b. Prediction of olive oil sensory descriptors using instrumental data fusion and partial least squares (PLS) regression. Talanta 155, 116-123.

Borràs, E., Mestres, M., Aceña, L., Busto, O., Ferré, J., Boqué, R., Calvo, A., 2015. Identification of olive oil sensory defects by multivariate analysis of mid infrared spectra. Food Chem. 187, 197-203.

Cadima, J., Cerdeira, J.O., Minhoto, M., 2004. Computational aspects of algorithms for variable selection in the context of principal components. Comput. Stat. Data Anal. 47, 225-236.

Cadima, J., Cerdeira, J.O., Silva, P.D., Minhoto, M., 2012. The subselect R package, $<$ http://cran.rproject.org/web/packages/subselect/vignettes/subselect. pdf $>$ (accessed 15.02.16)

Cayuela, J.A., Gomez-Coca, R.B., Perez-Camino, M.C., 2015. Sensory defects of virgi olive oil from a microbiological perspective. Trends Food Sci. Technol. 43, 227-235.

De Melo Milanez, K.D.T., Pontes, M.J.C., 2015. Classification of extra virgin olive oil and verification of adulteration using digital images and discriminant analysis. Anal. Methods 7, 8839-8846.

Di Serio, M.G., Giansante, L., Di Loreto, G., Faberi, A., Ricchetti, L., Di Giacinto, L., 2017. Ethyl esters versus fermentative organoleptic defects in virgin olive oil. Food Chem. $219,33-39$.

Dias, L.G., Rodrigues, N., Veloso, A.C.A., Pereira, J.A., Peres, A.M., 2016. Monovarieta extra virgin olive oils classification: a fusion of human sensory attributes and an electronic tongue. Eur. Food Res. Technol. 242, 259-270.

Dias, L.G., Fernandes, A., Veloso, A.C.A., Machado, A.A.S.C., Pereira, J.A., Peres, A.M., 2014. Single-cultivar extra virgin olive oil classification using a potentiometric electronic tongue. Food Chem. 160, 321-329.

Dias, L.G., Peres, A.M., Veloso, A.C.A., Reis, F.S., Vilas Boas, M., Machado, A.A.S.C., 2009 An electronic tongue taste evaluation: identification goat milk adulterations with bovine milk. Sens Actuat. B Chem. 136, 209-217.

Dias, L.G., Veloso, A.C.A., Sousa, M.E.B.C., Estevinho, L., Machado, A.A.S.C., Peres, A.M., 2015. A novel approach for honey pollen profile assessment using an electronic tongue and chemometric tools. Anal. Chim. Acta 900, 36-45.

EU No 1348/2013, 2013. Commission implementing regulation of 16 December 2013 amending Regulation No 2568/91/EEC on the characteristics of olive oil and oliveresidue oil and on the relevant methods of analysis. Off. J. Eur. Union L338, 31-67.

EU No 61/2011, 2011. Commission Regulation of 24 January 2011 amending Regulation No 2568/91/EEC on the characteristics of olive oil and olive pomace oil and on the relevant methods of analysis, Off. J. Eur. Union L23, pp. 1-13.

Farley III, C., Kassu, A., Bose, N., Jackson-Davis, A., Boateng, J., Ruffin, P., Sharma, A., 2017. Short distance standoff raman detection of extra virgin olive oil adulterated with canola and grapeseed oils. Appl. Spectrosc. 71, 1340-1347.

Georgouli, K., Del Rincon, J.M., Koidis, A., 2017. Continuous statistical modelling for rapid detection of adulteration of extra virgin olive oil using mid infrared and Raman spectroscopic data. Food Chem. 217, 735-742.

Hayashi, K., Matsuki, Y., Toko, K., Murata, T., Yamafuji, Ke., Yamafuji, Ka., 1989. Sensing of 'umami' taste and synergistic effects with synthetic lipid membranes. Sens. Mater. $1,321-334$.

Iiyama, S., Toko, K., Yamafuji, K., 1986. Effect of bitter substances on a model membrane system of taste reception. Agric. Biol. Chem. 50, 2709-2714.

IOC, Intliyama S., Toko K. and Yamafuji K., Effect of bitter substances on a model membrane system of taste reception, Agric, Biol. Chem.50, 1986, 2709-2714.[SPACE] ernational Olive Council, 2013. Sensory analysis of olive oil - Method for the organoleptic assessment of virgin olive oil. COI/T.20/Doc. No 15/Rev. 6 November 2013, 18pp. (http://www.internationaloliveoil.org/).

IOC, International Olive Council, 2014. IOC Mario Solinas quality award - Rules of the International competition for extra virgin olive oils. T.30/Doc. No 17 June 2014, 9pp. (http://www.internationaloliveoil.org/).

Jabeur, H., Zribi, A., Bouaziz, M., 2016. Extra-virgin olive oil and cheap vegetable oils: distinction and detection of adulteration as determined by GC and chemometrics. Food Anal. Method. 9, 712-723.

Jergović, A.-M., Peršurić, Ž., Saftić, L., Pavelić, S.K., 2017. Evaluation of MALDI-TOF/MS technology in olive oil adulteration. J. Am. Oil Chem. Soc. 94, 749-757.

Jolayemi, O.S., Tokatli, F., Buratti, S., Alamprese, C., 2017. Discriminative capacities of infrared spectroscopy and e-nose on Turkish olive oils. Eur. Food Res. Technol. 243, 2035-2042.

Kalaitzis, P., El-Zein, Z., 2016. Olive oil authentication, traceability and adulteration detection using DNA-based approaches. Lipid Technol. 28, 173-176.

Kirkpatrick, S., Gelatt, C.D., Vecchi, M.P., 1983. Optimization by simulated annealing. Science 220, 671-680.

Kobayashi, Y., Habara, M., Ikezazki, H., Chen, R., Naito, Y., Toko, K., 2010. Advanced taste sensors based on artificial lipids with global selectivity to basic taste qualities and high correlation to sensory scores. Sensors 10, 3411-3443.

Kuhn, M., Johnson, K., 2013. Applied Predictive Modeling. Springer Science Business Media, New York.

Kurihara, K., Yoshii, K., Kashiwayanagi, M., 1986. Transducers mechanisms in chemoreception. Comp. Biochem. Physiol. 85A, 1-22.
Lerma-García, M.J., Cerretani, L., Cevoli, C., Simó-Alfonso, E.F., Bendini, A., Toschi, T.G., 2010. Use of electronic nose to determine defect percentage in oils. Comparison with sensory panel results. Sens Actuat. B Chem. 147, 283-289.

Liu, L., Hu, C., Liu, L., Zhang, S., Chen, K., He, D., 2017. Rapid detection and separation of olive oil and Camellia oil based on ion mobility spectrometry fingerprints and chemometric models. Eur. J. Lipid Sci. Technol. 119, 1500463.

Marx, Í., Rodrigues, N., Dias, L.G., Veloso, A.C.A., Pereira, J.A., Drunkler, D.A., Peres, A.M., 2017a. Sensory classification of table olives using an electronic tongue: analysis of aqueous pastes and brines. Talanta 162, 98-106.

Marx, Í.M.G., Rodrigues, N., Dias, L.G., Veloso, A.C.A., Pereira, J.A., Drunkler, D.A., Peres, A.M., 2017b. Quantification of table olives' acid, bitter and salty tastes using potentiometric electronic tongue fingerprints. LWT Food Sci. Technol. 79, 394-401.

Marx, Í.M.G., Rodrigues, N., Dias, L.G., Veloso, A.C.A., Pereira, J.A., Drunkler, D.A., Peres, A.M., 2017c. Assessment of table olives' organoleptic defects intensities based on the potentiometric fingerprint recorded by an electronic tongue. Food Bioprocess Technol. 10, 1310-1323.

Mildner-Szkudlarz, S., Jeleń, H.H., 2008. The potential of different techniques for volatile compounds analysis coupled with PCA for the detection of the adulteration of olive oil with hazelnut oil. Food Chem. 110, 751-761.

Mildner-Szkudlarz, S., Jeleń, H.H., 2010. Detection of olive oil adulteration with rapeseed and sunflower oils using MOS electronic nose and SMPE-MS. J. Food Qual. 33, 21-41.

Morales, M.T., Luna, G., Aparicio, R., 2005. Comparative study of virgin olive oil sensory defects. Food Chem. 91, 293-301.

Mu, T., Chen, S., Zhang, Y., Chen, H., Guo, P., Meng, F., 2016. Portable Detection and Quantification of Olive Oil Adulteration by 473-nm Laser-Induced Fluorescence. Food Anal. Method. 9, 275-279.

Nigri, S., Oumeddour, R., 2016. Detection of extra virgin olive oil adulteration using Fourier transform infrared, synchronous fluorescence spectroscopy and multivariate analysis. Riv. Ital. Sostanze Gr. 93, 125-131.

Ok, S., 2017. Detection of olive oil adulteration by low-field NMR relaxometry and UV-Vis spectroscopy upon mixing olive oil with various edible oils. Grasas Aceites 68, e173.

Oliveros, M.C.C., Pavón, J.L.P., Pinto, C.G., Laespada, M.E.F., Cordero, B.M., Forina, M., 2002. Electronic nose based on metal oxide semiconductor sensors as a fast alternative for the detection of adulteration of virgin olive oils. Anal. Chim. Acta 459, 219-228.

Ou, G., Hu, R., Zhang, L., Li, P., Luo, X., Zhang, Z., 2015. Advanced detection methods for traceability of origin and authenticity of olive oils. Anal. Methods 7, 5731-5739.

Peris, M., Escuder-Gilabert, L., 2016. Electronic noses and tongues to assess food authenticity and adulteration. Trends Food Sci. Technol. 58, 40-54.

Philippidis, A., Poulakis, E., Papadaki, A., Velegrakis, M., 2017. Comparative study using Raman and visible spectroscopy of cretan extra virgin olive oil adulteration with sunflower oil. Anal. Lett. 50, 1182-1195.

Rashvand, M., Omid, M., Mobli, H., Firouz, M.S., 2016. Adulteration detection in olive oil using dielectric technique and data mining. Sens. Bio-Sens. Res. 11, 33-36.

Rodrigues, N., Dias, L.G., Veloso, A.C.A., Pereira, J.A., Peres, A.M., 2016. Monitoring olive oils quality and oxidative resistance during storage using an electronic tongue. LWT Food Sci. Technol. 73, 683-692.

Santonico, M., Grasso, S., Genova, F., Zompanti, A., Parente, F.R., Pennazza, G., 2015 Unmasking of olive oil adulteration via a multi-sensor platform. Sensors 15 21660-21672.

Santos, P.M., Kock, F.V.C., Santos, M.S., Lobo, C.M.S., Carvalho, A.S., Colnago, L.A., 2017. Non-invasive detection of adulterated olive oil in full bottles using time-domain NMR relaxometry. J. Brazil Chem. Soc. 28, 385-390.

Slim, S., Rodrigues, N., Dias, L.G., Veloso, A.C.A., Pereira, J.A., Oueslati, S., Peres, A.M., 2017. Application of an electronic tongue for Tunisian olive oils' classification according to olive cultivar or physicochemical parameters. Eur. Food Res. Technol. 243, $1459-1470$.

Souayah, F., Rodrigues, N., Veloso, A.C.A., Dias, L.G., Pereira, J.A., Oueslati, S., Peres, A.M., 2017. Discrimination of olive oil by cultivar, geographical origin and quality using potentiometric electronic tongue fingerprints. J. Am. Oil Chem. Soc. 94, 1417-1429.

Srigley, C.T., Oles, C.J., Kia, A.R.F., Mossoba, M.M., 2016. Authenticity assessment of extra virgin olive oil: evaluation of desmethylsterols and triterpene dialcohols. J. Am. Oil Chem. Soc. 93, 171-181.

Sun, X., Lin, W., Li, X., Shen, Q., Luo, H., 2015. Detection and quantification of extra virgin olive oil adulteration with edible oils by FT-IR spectroscopy and chemometrics. Anal. Methods 7, 3939-3945.

Uncu, A.T., Uncu, A.O., Frary, A., Doganlar, S., 2017. Barcode DNA length polymorphisms vs fatty acid profiling for adulteration detection in olive oil. Food Chem. 221, 1026-1033.

Valli, E., Bendini, A., Berardinelli, A., Ragni, L., Riccó, B., Grossi, M., Toschi, T.G., 2016. Rapid and innovative instrumental approaches for quality and authenticity of olive oils. Eur. J. Lipid Sci. Technol. 118, 1601-1619.

Veloso, A.C.A., Silva, L.M., Rodrigues, N., Rebello, L.P.G., Dias, L.G., Pereira, J.A., Peres, A.M., 2018. Perception of olive oils sensory defects using a potentiometric taste device. Talanta 176, 610-618.

Veloso, A.C.A., Dias, L.G., Rodrigues, N., Pereira, J.A., Peres, A.M., 2016. Sensory intensity assessment of olive oils using an electronic tongue. Talanta 146, 585-593.

Venables, W.N., Ripley, B.D., 2002. Modern Applied Statistics With S (Statistics and Computing), fourth ed. Springer, New York. 